\title{
Does Influenza A Infection Increase Oxidative Damage?
}

\author{
Mary P.E. Ng, Jetty C.Y. Lee, ${ }^{2}$ Wai Mun Loke, ${ }^{3}$ Leonard L.L. Yeo, ${ }^{4}$ Amy M.L. Quek, ${ }^{4}$ \\ Erle C.H. Lim, ${ }^{1,4}$ Barry Halliwell, ${ }^{5}$ and Raymond Chee-Seong Seet ${ }^{1,4}$
}

\begin{abstract}
Considerable data implicate oxidative damage in influenza pathogenesis. We examined temporal changes in oxidative damage using accurate biomarkers in an adult cohort with acute influenza infection and their relationships with clinical parameters. Clinical information and blood samples were collected during their acute illness and 3 months later. A fatigue questionnaire was administered 3 months following influenza infection. Thirty-five patients (mean age, 34 years) with polymerase chain reaction-confirmed influenza A infection were included; all patients returned for follow-up assessments. Adjusted levels of plasma $\mathrm{F}_{2}$-isoprostanes, total hydroxyeicosatetraenoic products (HETEs), $7 \beta$-hydroxycholesterol and 7-ketocholesterol, serum gamma-glutamyltransferase, and high-sensitivity C-reactive protein (hsCRP) were increased during the acute illness compared with age-matched controls. Despite clinical recovery, levels of these biomarkers remained higher at month 3 compared with controls. A proportion of patients had persistent symptoms such as fatigue (23\%), myalgia (14\%), and arthralgia (11\%) at month 3 . Patients with significant fatigue had higher baseline levels of plasma $\mathrm{F}_{2}$-isoprostanes, $\mathrm{F}_{4}$-neuroprostanes, and total HETEs compared to those without fatigue. By contrast, patients with persistent arthralgia and myalgia had higher baseline levels of serum hsCRP compared to those without these symptoms. Our observations lead to the hypothesis that oxidative damage participates in the pathogenesis of influenza infection and postinfectious fatigue. Antioxid. Redox Signal. 21, 1025-1031.
\end{abstract}

\section{Introduction}

Some evidence suggests a role for reactive oxygen spe$N_{\text {cies }}$ ROS) in influenza pathogenesis. In humans, the involvement of oxidative damage is suggested by lower plasma levels of antioxidants in children with influenza infection (3). Autopsy analysis of lung tissues from patients with influenza A pneumonia showed intense staining for oxidized dihydroethidium, indicating increased generation of oxygen radicals during severe infection (5). In healthy individuals, a challenge with live attenuated influenza vaccine elicited a prompt and sustained increase in biomarkers of oxidative stress in the breath (6). Available studies, however, have not evaluated the temporal changes of oxidative damage in influenza patients using accurate biomarkers. It is also

\section{Innovation}

Oxidative damage is increased during acute infection and is persistently elevated despite clinical recovery from influenza infection. Despite a relatively mild illness, this study revealed a proportion of patients with persistent symptoms of fatigue, myalgia, and arthralgia late into the disease, beyond the initial clinical recovery. Our observations lead to the hypothesis that oxidative damage is involved in the pathogenesis of influenza infection and postinfectious fatigue. These findings have important implications in the design of clinical trials investigating the roles of antioxidants in influenza infection.

\footnotetext{
${ }^{1}$ Department of Medicine, Yong Loo Lin School of Medicine, National University of Singapore, Singapore, Singapore.

${ }^{2}$ School of Biological Sciences, University of Hong Kong, Hong Kong, China.

${ }^{3}$ School of Chemical and Life Sciences, Nanyang Polytechnic, Singapore, Singapore.

${ }^{4}$ Department of Medicine, National University Health System, Singapore, Singapore.

${ }^{5}$ Department of Biochemistry, Yong Loo Lin School of Medicine, National University of Singapore, Singapore, Singapore.
} 
unclear whether oxidative damage is involved in the development of postinfluenza fatigue.

We prospectively investigated the temporal changes in oxidative damage among patients with influenza infection and compared them with clinical outcomes. Biomarkers that target different aspects of oxidative damage [including $\mathrm{F}_{2}$-isoprostanes, $\mathrm{F}_{4}$-neuroprostanes, cholesterol oxidation products (COPs), allantoin, and gamma-glutamyltransferase (GGT) (4)], were measured. We also examined total hydroxyeicosatetraenoic products (HETEs), which are potent inflammatory mediators, and the high-sensitivity C-reactive protein (hsCRP).

\section{Results}

\section{Participants}

All subjects were diagnosed by the attending physician to have acute influenza A infection, confirmed from nasopharyngeal swabs by real-time polymerase chain reaction. Thirty-five patients (mean age 34, range 21-63 years; 66\% men) were recruited and reassessed 3 months later. Seventy age-matched subjects were recruited as controls. The clinical

Table 1. Clinical and Laboratory Parameters

\begin{tabular}{|c|c|c|}
\hline Clinical information & $\begin{array}{l}\text { Acute illness } \\
\quad(\mathrm{n}=35)\end{array}$ & $\begin{array}{c}\text { Month } 3 \\
(\mathrm{n}=35)\end{array}$ \\
\hline Age (years) & $34.1(12.3)$ & \\
\hline \multicolumn{3}{|l|}{$\operatorname{Sex}(\%)$} \\
\hline Male & $23(66)$ & \\
\hline Female & $12(34)$ & \\
\hline Weight (kg) & $69.8(14.7)$ & $70.7(14.7)$ \\
\hline Body mass index $\left(\mathrm{kg} / \mathrm{m}^{2}\right)$ & $24.1(5.8)$ & $25.3(4.7)$ \\
\hline $\begin{array}{l}\text { Systolic blood pressure } \\
(\mathrm{mmHg})^{\mathrm{a}}\end{array}$ & $135(18)$ & $124(14)$ \\
\hline $\begin{array}{l}\text { Diastolic blood pressure } \\
(\mathrm{mmHg})^{\mathrm{a}}\end{array}$ & $83(11)$ & $77(11)$ \\
\hline Body temperature $\left({ }^{\circ} \mathrm{C}\right)^{\mathrm{a}}$ & $37.5(0.9)$ & $36.4(0.4)$ \\
\hline \multicolumn{3}{|l|}{ Risk factors $(\%)$} \\
\hline Asthma & $10(29)$ & \\
\hline Hypertension & $6(17)$ & \\
\hline Cigarette smoking & $6(17)$ & \\
\hline Hyperlipidemia & $5(14)$ & \\
\hline Ischemic heart disease & $3(9)$ & \\
\hline Diabetes mellitus & $2(6)$ & \\
\hline \multicolumn{3}{|l|}{ Laboratory results } \\
\hline \multicolumn{3}{|l|}{ Lipid profile } \\
\hline Triglycerides $(\mathrm{m} M)$ & $1.5(1.1)$ & $1.6(0.8)$ \\
\hline $\begin{array}{l}\text { High-density lipoprotein } \\
(\mathrm{m} M)\end{array}$ & $1.2(0.4)$ & $1.2(0.4)$ \\
\hline $\begin{array}{l}\text { Low-density lipoprotein } \\
\qquad(\mathrm{m} M)\end{array}$ & $3.2(1.0)$ & $3.2(1.1)$ \\
\hline Creatinine $(\mu M)$ & $90.3(77.2)$ & $85.2(76.7)$ \\
\hline Glucose $(\mathrm{m} M)$ & $5.2(1.9)$ & $5.5(2.4)$ \\
\hline Urea $(\mathrm{m} M)$ & $4.4(2.6)$ & $4.9(2.8)$ \\
\hline Alkaline phosphatase (U/L) & 79 (69-92) & $76(58-89)$ \\
\hline $\begin{array}{l}\text { Alkaline transaminases } \\
\text { (U/L) }\end{array}$ & $30(17-43)$ & $29(18-48)$ \\
\hline $\begin{array}{l}\text { Aspartate transaminases } \\
(\mathrm{U} / \mathrm{L})\end{array}$ & $29(23-40)$ & $27(21-37)$ \\
\hline
\end{tabular}

Values are expressed as mean (standard deviation) or median (interquartile range).

${ }^{\mathrm{a}} p<0.05$. characteristics of these patients are summarized in Table 1. Noteworthy is the increase in blood pressure parameters during acute illness compared with levels 3 months later. Cases and controls were comparable in terms of gender, body mass index, and medical comorbidities (data not shown). During acute illness, the mean sum of symptom score was 8.3; this mean score decreased to 0.5 three months later $(p<0.0001)$. Symptoms such as fever $(97 \%)$, headache $(86 \%)$, poor appetite $(63 \%)$, myalgia $(49 \%)$, and arthralgia (46\%), were common (Table 2). Three months after their acute illness, nine (26\%) subjects had persistent symptoms despite resolution of their initial symptoms. Significant fatigue was observed in eight (23\%) subjects, whereas a proportion had persistent musculoskeletal symptoms such as myalgia (14\%) and arthralgia (11\%) (Table 2). None of the controls reported any symptoms.

\section{Effects of influenza infection on biomarkers}

Lipid oxidation products. Compared with controls, plasma arachidonate levels of patients with influenza infection were lower during acute illness and 3 months later (Fig. 1A). Although serum cholesterol levels were lower during acute illness compared with controls, there were no differences in cholesterol levels compared with month 3 (Fig. 1B). By contrast, plasma docosahexaenoate levels were higher during acute illness and at month 3 compared with controls; higher levels were observed at month 3 compared with those during acute illness (Fig. 1C). Given these differences, lipid oxidation products are presented per unit of their respective precursors.

Plasma $\mathrm{F}_{2}$-isoprostanes (before and after adjusting for arachidonate) were higher during acute illness compared with levels at month 3 and those of controls (Fig. 2A, B). A nonsignificant increase in urinary $\mathrm{F}_{2}$-isoprostanes (per unit creatinine) was observed during acute illness compared with controls; urinary $\mathrm{F}_{2}$-isoprostanes (per unit creatinine) tended to be higher at month 3 compared with during the acute illness. Despite clinical recovery 3 months later, plasma and urinary levels of $\mathrm{F}_{2}$-isoprostanes (per unit creatinine) were persistently higher than control levels (Fig. 2A-C). Plasma $7 \beta$-hydroxycholesterol and 7-ketocholesterol (per unit cholesterol) were significantly increased during acute illness

Table 2. Symptom Frequency

\begin{tabular}{|c|c|c|c|c|}
\hline \multirow[b]{2}{*}{ Symptoms } & \multicolumn{2}{|c|}{ Acute illness (\%) } & \multicolumn{2}{|c|}{ Month $3(\%)$} \\
\hline & Score 0 & Score 1-3 & Score 0 & Score 1-3 \\
\hline Fever & $1(3)$ & $34(97)$ & $35(100)$ & $0(0)$ \\
\hline $\begin{array}{c}\text { Nausea and } \\
\text { vomiting }\end{array}$ & $21(60)$ & $14(40)$ & $34(97)$ & $1(3)$ \\
\hline $\begin{array}{l}\text { Abdominal } \\
\text { pain }\end{array}$ & $24(69)$ & $11(31)$ & 35 (100) & $0(0)$ \\
\hline Diarrhea & $26(74)$ & $9(26)$ & $35(100)$ & $0(0)$ \\
\hline Poor appetite & $13(37)$ & $22(63)$ & $33(94)$ & $2(6)$ \\
\hline Headaches & $5(14)$ & $30(86)$ & $32(91)$ & $3(9)$ \\
\hline Arthralgia & $19(54)$ & $16(46)$ & 31 (89) & $4(11)$ \\
\hline Myalgia & $18(51)$ & 17 (49) & $30(86)$ & $5(14)$ \\
\hline $\begin{array}{l}\text { Total } \\
\text { symptom } \\
\text { score }\end{array}$ & $0(0)$ & $35(100)$ & $26(74)$ & $9(26)$ \\
\hline
\end{tabular}



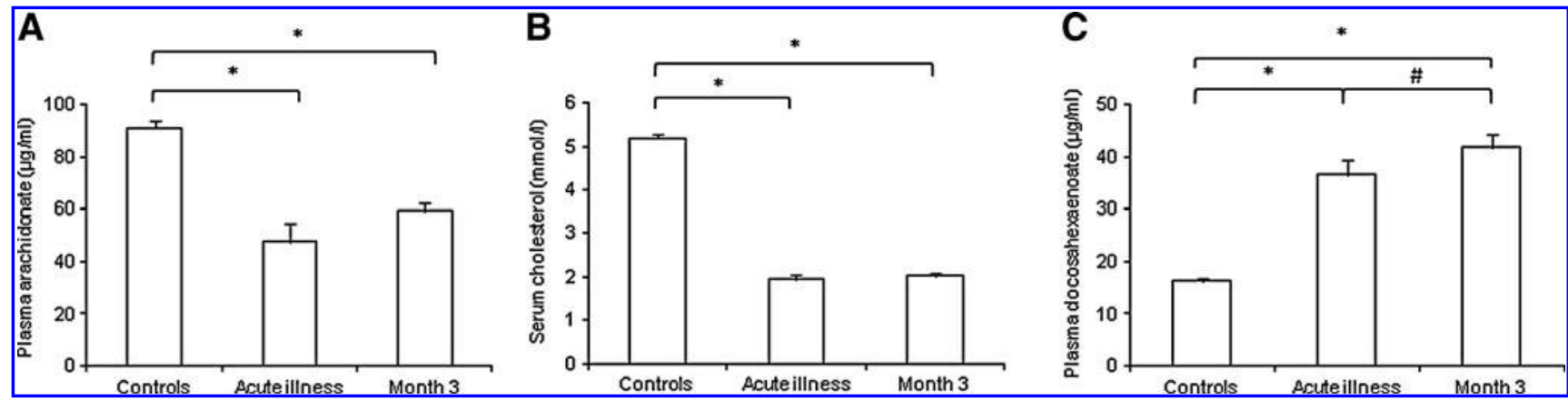

FIG. 1. Comparisons in the levels of (A) plasma arachidonate, (B) serum cholesterol, and (C) plasma docosahexaenoate in age-matched controls $(n=70)$ and influenza patients $(n=35)$. Each graphical column expresses mean \pm standard deviation. ${ }^{*} p<0.05$ using the unpaired Student's $t$-test versus controls, while ${ }^{\#} p<0.05$ using the paired $t$ test between acute illness and at month 3 .

compared with levels of controls and those 3 months later (Fig. 3A, B). Similarly, plasma $7 \beta$-hydroxycholesterol and 7-ketocholesterol (per unit cholesterol) did not normalize and remained higher than controls 3 months later. Conversely, plasma $\mathrm{F}_{4}$-neuroprostanes (before and after adjusting for docosahexaenoate) were lower during the acute illness and at month 3 as compared with controls; levels measured during acute illness were higher compared with those at month 3 (Fig. 3C, D).

Urate, allantoin, and GGT. During acute illness, plasma urate was significantly lower than controls; these levels increased at month 3, but remained lower compared with controls (Fig. 4A). By contrast, plasma allantoin was higher during acute illness compared with controls and increased further at month 3 (Fig. 4B). Serum GGT was significantly increased during acute illness compared with controls (Fig. 4C). Although serum GGT trended lower at month 3, GGT levels remained higher than controls.

Total HETEs, hsCRP, nitrite, and nitrate. Plasma total HETEs (per unit arachidonate) and serum hsCRP levels were higher during acute illness compared with controls (Fig. 5A, B). Although levels were lower at month 3 compared with during acute illness, these biomarkers did not normalize to levels of controls. By comparison, plasma nitrite and nitrate were lower during acute illness compared with controls, these levels increased significantly at month 3. Notably, levels of plasma nitrite were higher at month 3 compared with controls (Fig. 5C, D).

\section{Correlations between biomarkers and clinical parameters}

Significant correlation was observed between age and plasma docosahexaenoate (acute illness: $r=0.43, p=0.01$; month 3: $r=0.41, p=0.01$ ), but not with the other measured biomarkers. No differences in biomarkers were observed between genders. Plasma nitrite was observed to correlate positively with systolic blood pressure during acute illness $(r=0.38, p=0.029)$; this relationship, however, was not seen 3 months later. No significant correlation was found between systolic and diastolic blood pressure and plasma nitrate, arachidonate, $\mathrm{F}_{2}$-isoprostanes, total HETEs, $\mathrm{F}_{4}$-neuroprostanes, COPs, and allantoin during acute illness and 3 months later. Similarly, there was no correlation between body weight, height, body mass index, and smoking status and the measured biomarkers.

\section{Comparisons between biomarkers and clinical features}

During acute illness and at month 3 , there were no significant correlations between biomarker levels and symptom scores. Post hoc analyses were performed to examine the relationship between levels of biomarkers among subjects with and without significant fatigue, myalgia, and arthralgia.

Patients with significant fatigue had higher acute levels of plasma $\mathrm{F}_{2}$-isoprostanes, $\mathrm{F}_{4}$-neuroprostanes, total HETEs,


FIG. 2. Comparisons in the (A) unadjusted levels of plasma $F_{2}$-isoprostanes, (B) adjusted levels of plasma $F_{2}$ isoprostanes, and $(\mathbf{C})$ urinary $\mathbf{F}_{2}$-isoprostanes between age-matched controls and patients during acute illness and at month 3. Each graphical column expresses mean \pm standard deviation. ${ }^{*} p<0.05$ using the unpaired Student's $t$-test versus controls, while ${ }_{p}^{\#}<0.05$ using the paired $t$-test between acute illness and at month 3 . 


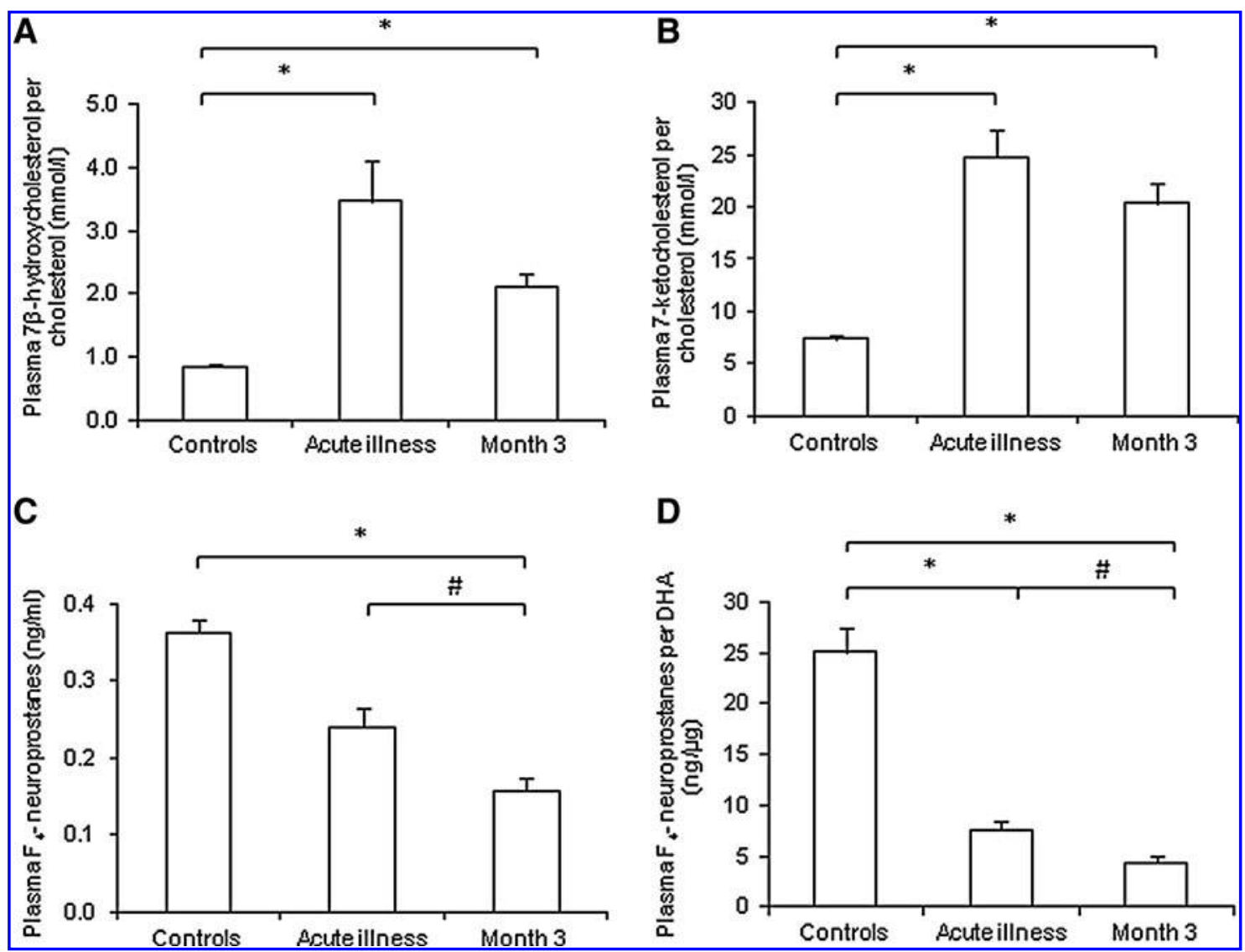

FIG. 3. Comparisons in the adjusted levels of (A) plasma 7 $\beta$-hydroxycholesterol, (B) plasma 7-ketocholesterol, (C) unadjusted plasma $F_{4}$-neuroprostanes, and (D) adjusted plasma $F_{4}$-neuroprostanes between age-matched controls and patients during acute illness and at month 3 . Each graphical column expresses mean \pm standard deviation. $* p<0.05$ using the unpaired Student's $t$-test versus controls, while ${ }^{\#} p<0.05$ using the paired $t$-test between acute illness and at month 3 .

and serum hsCRP compared to those without fatigue (Fig. 6A-D). No differences were observed between fatigue and arachidonate, docosahexaenoate, COPs, urate, nitrite, nitrate, and GGT. There were no differences in these biomarkers measured at month 3 between subjects with and without fatigue (data not shown). At month 3, subjects with persistent arthralgia and myalgia had higher acute levels of serum hsCRP compared with those without arthralgia and myalgia (Fig. 7A, B). No significant relationship was observed between biomarkers of oxidative damage and these musculoskeletal symptoms.

\section{Discussion and Future Directions}

Biomarkers of oxidative damage were rigorously assessed in patients with influenza infection. This study is the first to examine postinfectious fatigue in influenza patients and implicates oxidative damage in its pathogenesis. Despite
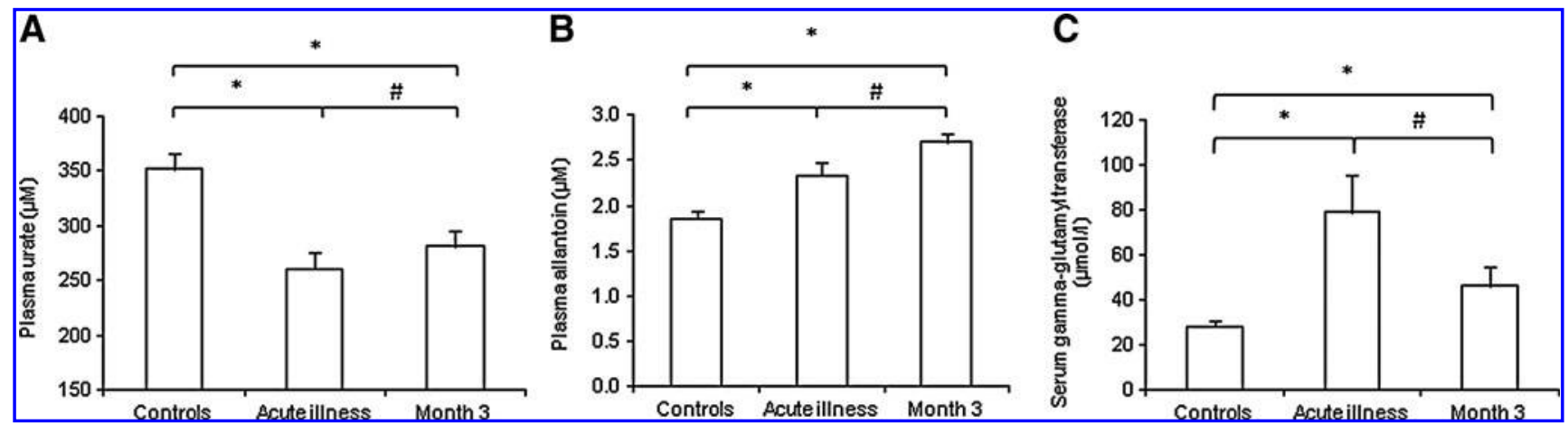

FIG. 4. Comparisons in the adjusted levels of (A) plasma urate, (B) plasma allantoin, and (C) serum gammaglutamyltransferase between age-matched controls and patients during acute illness and at month 3 . Each graphical column expresses mean \pm standard deviation. ${ }^{*} p<0.05$ using the unpaired Student's $t$-test versus controls, while ${ }^{\#} p<0.05$ using the paired $t$-test between acute illness and at month 3 . 


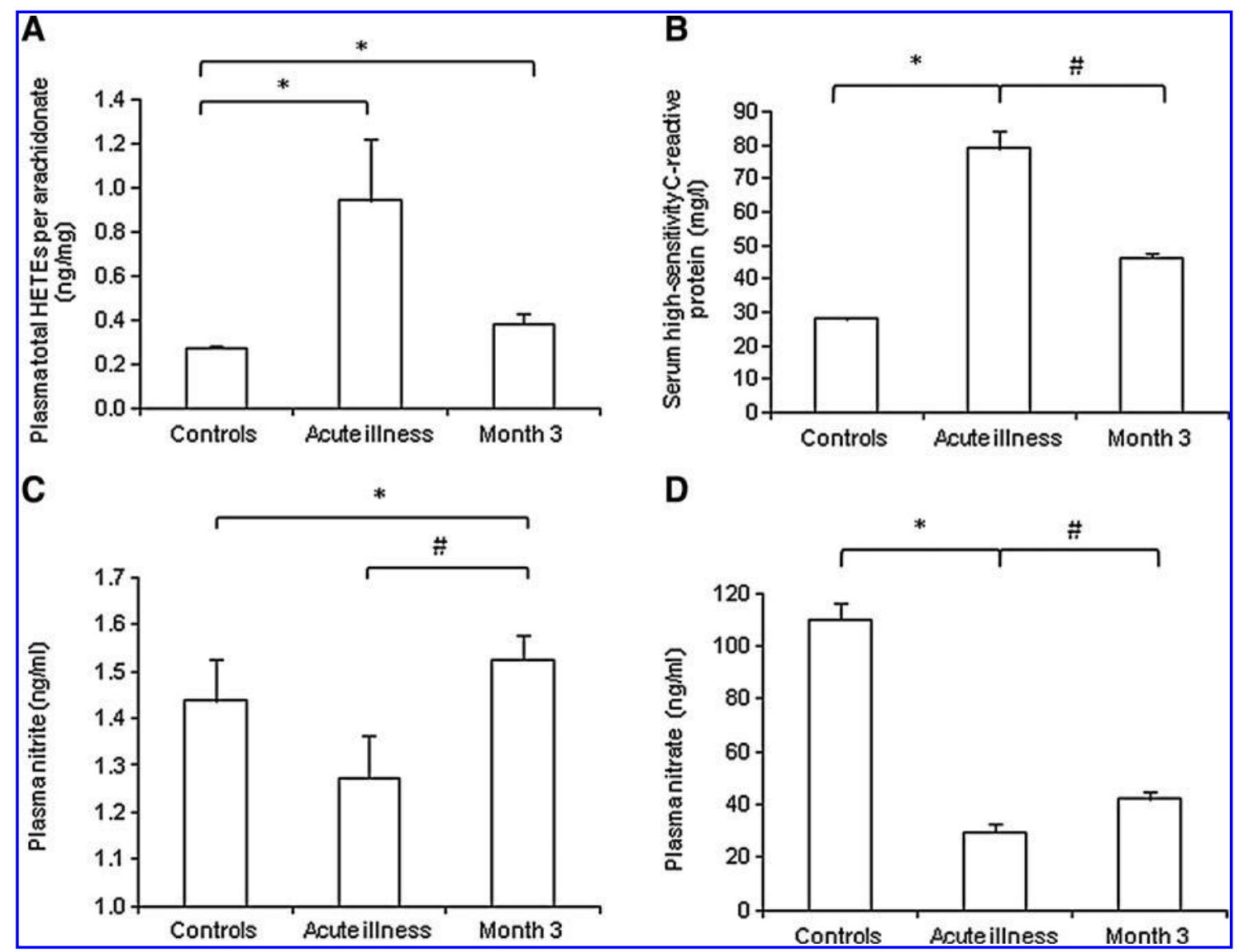

FIG. 5. Comparisons in the (A) adjusted levels of plasma total hydroxyeicosatetraenoic products (HETEs), (B) serum high-sensitivity C-reactive protein (hsCRP), (C) plasma nitrite, and (D) plasma nitrate between age-matched controls and patients during acute illness and at month 3 . Each graphical column expresses mean \pm standard deviation. $* p<0.05$ using the unpaired Student's $t$-test versus controls, while ${ }^{\#} p<0.05$ using the paired $t$-test between acute illness and at month 3 .

apparent clinical recovery, a proportion of patients had persistent elevation in these biomarkers compared with controls. The strengths of this study include the prospective study design, use of paired clinical and biomarker determination, and measurement of accurate biomarkers of oxidative damage.

Plasma $\mathrm{F}_{2}$-isoprostanes were observed to be higher during acute illness compared with recovery levels and those of controls. The increase in oxidative damage is also suggested by an increase in several biomarkers of lipid peroxidation such as $7 \beta$-hydroxycholesterol and 7-ketocholesterol. These biomarkers remain elevated despite clinical recovery 3 months later. Contrary to our previous findings in dengue patients (7), we did not observe an association between plasma total HETEs and blood pressure, possibly due to lack of vascular involvement in influenza infection. Increased vascular permeability, on the other hand, is central to the pathogenesis of dengue infection.

Levels of serum GGT peak during acute illness and fall (but not normalizing to control levels) at month 3 . Increase in oxidative damage is also supported by correspondingly lower levels of uric acid which, in vivo, has been suggested to be a potent antioxidant (1). Significant changes in plasma allantoin, a product of attack upon urate by ROS, were observed during acute infection. Even higher levels were seen at month 3 , indicative of ongoing oxidative damage, especially as al- lantoin levels seem to normalize quickly when oxidative stress ceases (8). We did not observe an increase in circulatory nitrite or nitrate during influenza infection. Instead, nitrate appeared to be lower during acute illness. Levels of both plasma nitrite and nitrate were lower during acute illness, suggesting decreased nitric oxide production and, consequently, endothelial dysfunction.

We observed higher baseline levels of plasma $\mathrm{F}_{2}$-isoprostanes and $\mathrm{F}_{4}$-neuroprostanes in patients with postinfectious fatigue compared with those without fatigue. By contrast, patients with musculoskeletal complaints such as myalgia and arthralgia had higher baseline hsCRP, but not biomarkers of oxidative damage. Together, these data suggest a modulatory role of ROS in immune activation, important both in the eradication of viral infection and immune-induced cellular injury.

\section{Notes}

\section{Study population}

Subjects attending the Emergency Room at the National University Hospital, Singapore, with influenza-like symptoms were screened. Recruitment criteria included the presence of fever and at least two of the following symptoms (cough, sore throat, rhinorrhea, and/or myalgia). Information on age, gender, clinical parameters, and comorbidities was collected after written informed consent was obtained. Each 


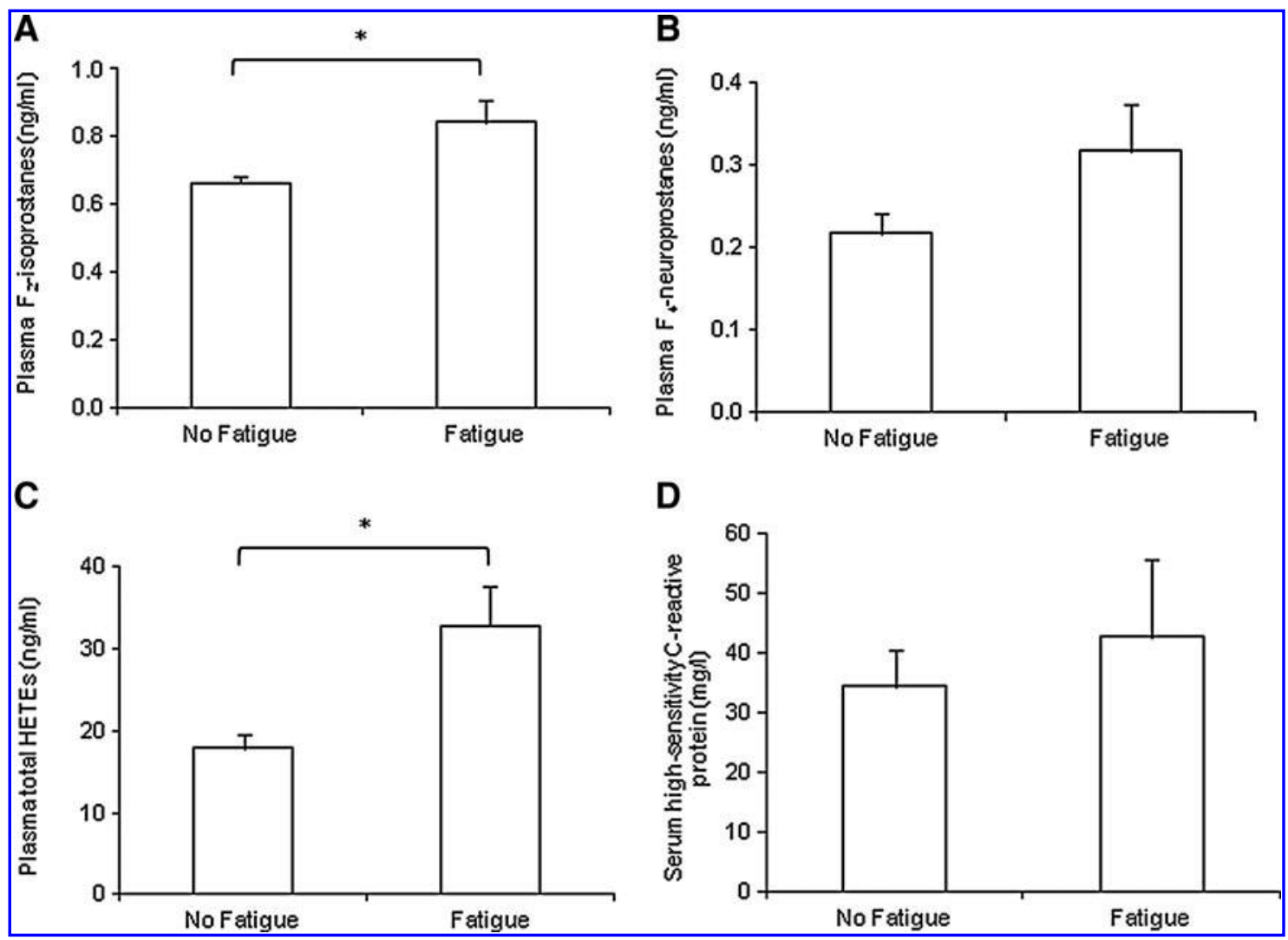

FIG. 6. Acute levels of (A) plasma $F_{2}$-isoprostanes, (B) plasma $F_{4}$-neuroprostanes, (C) plasma total HETEs, and (D) serum hsCRP in patients with or without postinfectious fatigue at month 3 . Each graphical column expresses mean \pm standard deviation. ${ }^{*} p<0.05$ using the unpaired Student's $t$-test versus controls.

subject was administered a detailed questionnaire to determine their symptoms and severity $(0=$ none; $1=$ mild; $2=$ moderate; $3=$ severe). The sum of these scores was used to indicate symptom severity.

Study subjects were invited to return 3 months later. During this review, they were administered the same questionnaire on symptom severity. The presence of fatigue was determined using a fatigue questionnaire (FQ). Briefly, the
FQ is a validated questionnaire, consisting of 11 items that measure the physical and mental dimensions of fatigue. Physical fatigue corresponds to the subjective feeling of being exhausted and lacking energy, whereas mental fatigue describes the subjective feeling of being mentally exhausted, incorporating items on concentration, memory, and speech. The seven items on physical fatigue and the four items on mental fatigue have four response categories $(0=$ none;

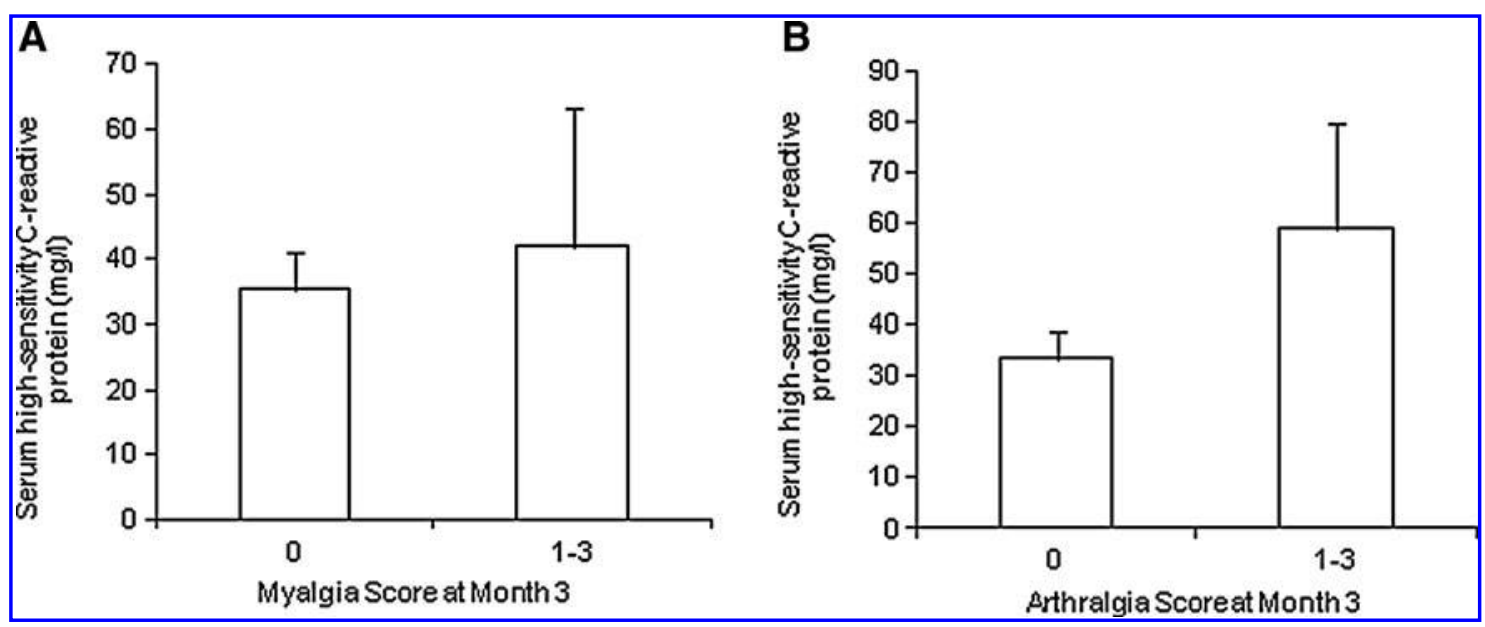

FIG. 7. Acute levels of serum hsCRP in patients with persistent (A) myalgia and (B) arthralgia at month 3 . Each graphical column expresses mean \pm standard deviation. 
$1=$ mild $; 2=$ moderate $3=$ severe $)$. Thus, higher scores imply more fatigue, with a maximum scale score of 33 . Fatigue is considered to be clinically significant when the fatigue score is $>4$ (2). Age- and gender-matched healthy volunteers were recruited to serve as study controls. The study protocol was reviewed and approved by the Domain Specific Review Board, National Healthcare Group, Singapore.

\section{Measurement of biomarkers}

Blood and urine samples were collected from patients into EDTA and plain tubes during each visit. Blood samples were centrifuged at $2800 \mathrm{rpm}$ for $15 \mathrm{~min}$ to derive plasma and serum. Indomethacin ( $3 \mu \mathrm{l} 5 \mathrm{~m} M$ to $1 \mathrm{ml}$ of plasma) and butylated hydroxytoluene ( $20 \mu \mathrm{l} 2 \mathrm{~m} M$ to $1 \mathrm{ml}$ of plasma) were added into plasma samples to prevent artifactual oxidation during storage at $-80^{\circ} \mathrm{C}$.

$\mathrm{F}_{2}$-isoprostanes, total HETEs, $\mathrm{F}_{4}$-neuroprostanes, COPs, allantoin, nitrite, and nitrate were measured in plasma by gas chromatography-mass spectrometry (Agilent Technologies). To take into account the precursor levels of these metabolites, arachidonate, docosahexaenoate, cholesterol, and urate levels were measured. Urinary $\mathrm{F}_{2}$-isoprostanes were expressed per unit creatinine. Low-density lipoprotein cholesterol and high-density lipoprotein cholesterol, hsCRP, creatinine, and glucose concentrations were measured in serum using the Cobas c111 Photometric Analyzer (Roche Diagnostic $\mathrm{GmbH}$ ).

\section{Statistical analyses}

Statistical analyses were performed using SPSS version 20.0 (SPSS, Inc.). Mean (standard deviation) was used to describe parametric data, while median (interquartile range) was used to describe nonparametric data. For normally distributed data, between-group (influenza $v s$. controls) and within-group (acute illness and at month 3) differences were analyzed using unpaired and paired $t$-tests, respectively. For nonparametric data, between-group and withingroup differences were analyzed using Mann-Whitney unpaired and Wilcoxon paired tests, respectively. Pearson correlation analysis was used to assess the relationships between various biomarkers. Statistical significance was set as $p<0.05$.

\section{Acknowledgments}

The authors thank the participants and the National Medical Research Council, Singapore (NMRC/CSA/030/2011 and NMRC/EDG/1050/2011) for their generous support.

\section{References}

1. Becker BF. Towards the physiological function of uric acid. Free Radic Biol Med 14: 615-631, 1993.
2. Chalder T, Berelowitz G, Pawlikowska T, Watts L, Wessely S, Wright D, and Wallace EP. Development of a fatigue scale. J Psychosom Res 37: 147-153, 1993.

3. Kelekci S, Evliyaoglu O, Sen V, Yolbas I, Uluca U, Tan I, and Gurkan MF. The relationships between clinical outcome and the levels of total antioxidant capacity (TAC) and coenzyme $\mathrm{Q}\left(\mathrm{CoQ}_{10}\right)$ in children with pandemic influenza (H1N1) and seasonal flu. Eur Rev Med Pharmacol Sci 16: 1033-1038, 2012.

4. Lee DH, Blomhoff R, and Jacobs DR, Jr. Is serum gamma glutamyltransferase a marker of oxidative stress? Free Radic Res 38: 535-539, 2004.

5. Nin N, Sanchez-Rodriguez C, Ver LS, Cardinal P, Ferruelo A, Soto L, Deicas A, Campos N, Rocha O, Ceraso DH, El-Assar M, Ortin J, Fernandez-Segoviano P, Esteban A, and Lorente JA. Lung histopathological findings in fatal pandemic influenza A (H1N1). Med Intensiva 36: 24 31, 2012.

6. Phillips M, Cataneo RN, Chaturvedi A, Danaher PJ, Devadiga A, Legendre DA, Nail KL, Schmitt P, and Wai J. Effect of influenza vaccination on oxidative stress products in breath. J Breath Res 4: 026001, 2010.

7. Seet RC, Lee CY, Lim EC, Quek AM, Yeo LL, Huang SH, and Halliwell B. Oxidative damage in dengue fever. Free Radic Biol Med 47: 375-380, 2009.

8. Seet RC, Lee CY, Loke WM, Huang SH, Huang H, Looi WF, Chew ES, Quek AM, Lim EC, and Halliwell B. Biomarkers of oxidative damage in cigarette smokers: which biomarkers might reflect acute versus chronic oxidative stress? Free Radic Biol Med 50: 1787-1793, 2011.

Address correspondence to: Dr. Raymond Chee-Seong Seet Department of Medicine National University Health System NUHS Tower Block

1 E Kent Ridge Road Singapore 119228 Singapore

E-mail: raymond_seet@nus.edu.sg

Date of first submission to ARS Central, March 21, 2014; date of acceptance, March 27, 2014.

$$
\begin{aligned}
& \text { Abbreviations Used } \\
\mathrm{COPs} & =\text { cholesterol oxidation products } \\
\mathrm{FQ} & =\text { fatigue questionnaire } \\
\mathrm{GGT} & =\text { gamma-glutamyltransferase } \\
\mathrm{HETES} & =\text { hydroxyeicosatetraenoic products } \\
\text { hSCRP } & =\text { high-sensitivity } \mathrm{C}-\text { reactive protein } \\
\mathrm{ROS} & =\text { reactive oxygen species }
\end{aligned}
$$


This article has been cited by:

1. K. S. Leung, J. M. Galano, T. Durand, J. C.-Y. Lee. 2014. Current development in non-enzymatic lipid peroxidation products, isoprostanoids and isofuranoids, in novel biological samples. Free Radical Research 1-11. [CrossRef]

2. Jean-Marie Galano, Jetty Chung-Yung Lee, Cecile Gladine, Blandine Comte, Jean-Yves Le Guennec, Camille Oger, Thierry Durand. 2014. Non-enzymatic cyclic oxygenated metabolites of adrenic, docosahexaenoic, eicosapentaenoic and $\alpha$-linolenic acids; bioactivities and potential use as biomarkers. Biochimica et Biophysica Acta (BBA) - Molecular and Cell Biology of Lipids . [CrossRef] 